essentials 
essentials liefern aktuelles Wissen in konzentrierter Form. Die Essenz dessen, worauf es als „State-of-the-Art“ in der gegenwärtigen Fachdiskussion oder in der Praxis ankommt. essentials informieren schnell, unkompliziert und verständlich

- als Einführung in ein aktuelles Thema aus Ihrem Fachgebiet

- als Einstieg in ein für Sie noch unbekanntes Themenfeld

- als Einblick, um zum Thema mitreden zu können

Die Bücher in elektronischer und gedruckter Form bringen das Expertenwissen von Springer-Fachautoren kompakt zur Darstellung. Sie sind besonders für die Nutzung als eBook auf Tablet-PCs, eBook-Readern und Smartphones geeignet. essentials: Wissensbausteine aus den Wirtschafts-, Sozial- und Geisteswissenschaften, aus Technik und Naturwissenschaften sowie aus Medizin, Psychologie und Gesundheitsberufen. Von renommierten Autoren aller Springer-Verlagsmarken.

Weitere Bände in dieser Reihe http://www.springer.com/series/13088 
Manuel Faßmann • Christoph Moss

\section{Instagram als \\ Marketing-Kanal}

Die Positionierung ausgewählter

Social-Media-Plattformen

Springer VS 
Manuel Faßmann

Agentur für Kommunikation

mediamoss $\mathrm{GmbH}$

Dortmund, Deutschland
Prof. Dr. Christoph Moss

Agentur für Kommunikation

mediamoss $\mathrm{GmbH}$

Dortmund, Deutschland
ISSN 2197-6708

essentials

ISBN 978-3-658-14348-0

DOI 10.1007/978-3-658-14349-7
ISSN 2197-6716 (electronic)

ISBN 978-3-658-14349-7 (eBook)

Die Deutsche Nationalbibliothek verzeichnet diese Publikation in der Deutschen Nationalbibliografie; detaillierte bibliografische Daten sind im Internet über http://dnb.d-nb.de abrufbar.

\section{Springer VS}

(C) Springer Fachmedien Wiesbaden 2016

Das Werk einschließlich aller seiner Teile ist urheberrechtlich geschützt. Jede Verwertung, die nicht ausdrücklich vom Urheberrechtsgesetz zugelassen ist, bedarf der vorherigen Zustimmung des Verlags. Das gilt insbesondere für Vervielfältigungen, Bearbeitungen, Übersetzungen, Mikroverfilmungen und die Einspeicherung und Verarbeitung in elektronischen Systemen.

Die Wiedergabe von Gebrauchsnamen, Handelsnamen, Warenbezeichnungen usw. in diesem Werk berechtigt auch ohne besondere Kennzeichnung nicht zu der Annahme, dass solche Namen im Sinne der Warenzeichen- und Markenschutz-Gesetzgebung als frei zu betrachten wären und daher von jedermann benutzt werden dürften.

Der Verlag, die Autoren und die Herausgeber gehen davon aus, dass die Angaben und Informationen in diesem Werk zum Zeitpunkt der Veröffentlichung vollständig und korrekt sind. Weder der Verlag noch die Autoren oder die Herausgeber übernehmen, ausdrücklich oder implizit, Gewähr für den Inhalt des Werkes, etwaige Fehler oder Äußerungen.

Gedruckt auf säurefreiem und chlorfrei gebleichtem Papier

Springer VS ist Teil von Springer Nature

Die eingetragene Gesellschaft ist Springer Fachmedien Wiesbaden GmbH 


\section{Was Sie in diesem essential finden können}

- Kenntnisse der Strukturen, des Aufbaus und Umgangs von und mit einer Marke

- Die Entwicklung von Instagram sowie die grundlegenden Strukturen der Fotound Videosharing Community

- Gründe, warum es sich bei Instagram um ein essenzielles Marketing Tool im Web 2.0 handelt

- Den Vergleich der Kerneigenschaften der Social-Media-Kanäle Instagram, Facebook und Twitter auf Basis ihrer Charakterisierungen durch Nutzer und Unternehmen

- Die Positionierung von Instagram, Facebook und Twitter im Social-Media-Marketing-Kommunikationsmix

- Handlungsempfehlungen, wofür Unternehmen die Foto- und Videosharing Community Instagram in Abgrenzung zu Facebook und Twitter strategisch einsetzen können 


\section{Vorwort}

Social Media ist das Schlagwort der vergangenen beiden Jahrzehnte. Menschliche Beziehungen verlagern sich zunehmend in das digitale Universum. Im Internet wird vernetzt, geteilt, gefolgt und abonniert. Nutzer diskutieren und entdecken neue Trends. Sie tauschen Gedanken aus und verursachen Shitstorms. Im Netz finden sich Nischengruppen, die sich in der realen Welt niemals gefunden hätten. Menschen lernen sich kennen, die sonst niemals ein Wort miteinander hätten wechseln können. Sei es auf Blogs, Microblogs, in sozialen Netzwerken oder auf Foto- und Videosharing-Plattformen - die sozialen Medien bieten ihren Nutzern vielfältige neue Arten, miteinander zu kommunizieren, und bestimmen einen großen Teil des heutigen Lebens. Und das Angebot wird ausgiebig genutzt: 56,1 Mio. Menschen in der deutschen Bevölkerung sind mittlerweile online (ardzdf-onlinestudie.de 2015). Und davon sind bereits $78 \%$ auf mindestens einer Social-Media-Plattform angemeldet (Bitkom 2013). Dabei entwickelt sich die Form und Idee der Nutzung der sozialen Medien stetig weiter.

Niemand hätte vor 15 Jahren geglaubt, dass Marketer einmal maßgeblich auf die Werbeformen im Internet - gar in den sozialen Medien - angewiesen sind. Wäre einem Menschen vor 15 Jahren erklärt worden, dass man kaum noch mit dem Handy oder Smartphone telefoniert, sondern vielmehr Zeit in den sozialen Medien damit verbringt - man hätte es wahrscheinlich noch weniger glauben können (Ambs und Schmied 2014).

Aber genau das ist heute Realität. Und eben dieses Potenzial bietet nicht nur den Menschen untereinander eine schier grenzenlose Kommunikationsfreiheit, sondern ist auch für Unternehmen und deren Marken eine bedeutende Chance, ihre Kommunikation neu zu erfinden. Bedingung dafür ist allerdings das Verständnis der Charakteristiken der verschiedenen sozialen Plattformen.

Instagram scheint den Nerv der Zeit getroffen zu haben und ist dabei, eine immer bedeutendere Rolle im Marketing-Mix einzunehmen. Die rasant 
wachsenden Nutzerzahlen und die jüngst erreichte Marke von 400 Mio. Usern sprechen für sich (Instagram 2016) und vor allem auch für Bilder, Emotionen und Storytelling. „Marken und Konsumenten standen sich noch nie so nah“ (Nieberding 2014), schreibt Nieberding im Jahr 2014 in Bezug auf Instagram in einem Artikel für Die Zeit. Inwieweit diese Behauptung zutrifft, ist Gegenstand der Untersuchungen dieses essentials.

Dortmund, Deutschland

Manuel Faßmann

Christoph Moss 


\section{Inhaltsverzeichnis}

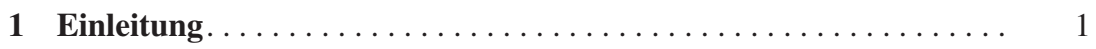

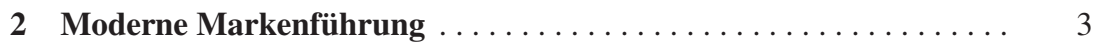

2.1 Markenidentität ........................... 3

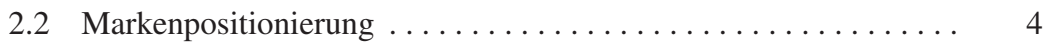

2.3 Markenpersönlichkeit........................ 5

2.4 Markenbeziehung. . . . . . . . . . . . . . . . . 7

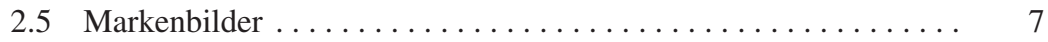

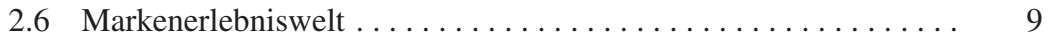

2.7 Markenästhetik.......................... 10

3 Instagram als Marketing-Kanal $\ldots \ldots \ldots \ldots \ldots \ldots \ldots \ldots \ldots$

3.1 Die grundlegenden Strukturen von Instagram ............. 13

3.2 Instagram als Marketing Tool. .................... 14

3.3 Die Relevanz von Instagram als Marketing-Kanal ........... 19

4 Charakterisierung und Positionierung der Social-Media-Plattformen ..................... 23

4.1 Der Untersuchungsvorgang $\ldots \ldots \ldots \ldots \ldots \ldots \ldots \ldots \ldots \ldots \ldots \ldots \ldots \ldots$

4.2 Positionierung und Charakterisierung von Instagram, Facebook und Twitter. . . . . . . 26

5 Fazit und Handlungsempfehlungen $\ldots \ldots \ldots \ldots \ldots \ldots \ldots \ldots \ldots \ldots \ldots \ldots$

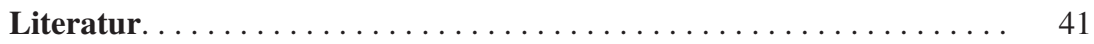

